\title{
Congenital tuberculosis with possible placental transmission and paradoxical reaction to anti-tuberculosis treatment
}

\author{
Lokesh Tiwari* and Rajesh Kumar \\ Department of Pediatrics, All India Institute of Medical Sciences Patna, India
}

\begin{abstract}
Congenital tuberculosis (CTB) is a rare disease with a high mortality rate, requiring high index of suspicion to diagnose because of non-specific presentation. This report describes a case of 6 month male child with congenital tuberculosis with possible placental transmission. Infant had fever, fast breathing and cough since 20 days of life. Ultrasonography (USG) abdomen showed granulomatous lesions in liver. Although mother was diagnosed to be having tuberculosis during $3^{\text {rd }}$ week after delivery and started on anti-tuberculosis treatment (ATT), child remained undiagnosed for 6 months and didn't get appropriate treatment. Fever, respiratory distress and cough initially improved after starting ATT but patient worsened after 2 weeks of ATT which could not be attributed to cause other than tuberculosis paradoxical reaction (TB-PR). Patient was given steroid following which he gradually improved. This case highlights the importance of keeping high index of suspicion for congenital TB in infants, especially in developing nations, and need for early diagnosis and treatment for the survival of theses infants. PR should be considered even in infancy if patient shows worsening of symptoms after initial improvement that couldn't be explained otherwise.
\end{abstract}

\section{Introduction}

Congenital tuberculosis is extremely rare condition with less than 400 cases reported in the literature [1] having a high mortality. Reported incidence is gradually declining significantly with only 18 cases been reported from 2001 to December 2005 [2]. Paradoxical reaction to ATT in case of congenital tuberculosis has been described only once in literature [3]. The importance of early diagnosis and treatment of CTB is crucial considering nonspecific nature of presentation and high mortality associated with it. Paradoxical reaction (PR) to ATT should be thought of if there is worsening of clinical or radiological findings following the initiation of appropriate antituberculosis treatment as early institution of steroid could be life saving in these children.

We report a case of congenital tuberculosis remaining undiagnosed for 6 months, till it presented to our institute, who when started on ATT after initial improvement, worsened and then responded favourably to steroid. A written consent was sought from the legal guardians of the index child to publish the findings, however ethical clearance was waived for this being a retrospective anonymous case report.

\section{Case}

A 6 month male child presented with history of fast breathing, cough, fever since 20 days of life. He was normal vaginal delivered at term, weighing $1.5 \mathrm{~kg}$. Child had received multiple antibiotics prior to presentation in our institute. During third week after delivery mother developed productive cough, fever and was diagnosed to be having tuberculosis (TB) and was started on ATT.

On examination he was sick looking, malnourished, weighing $3.24 \mathrm{~kg}$ ( $\mathrm{z}$ score $<-3.0)$ with length of $60 \mathrm{~cm}$ ( $\mathrm{z}$ score $<-3.0)$ and head circumference of $37 \mathrm{~cm}$ ( $\mathrm{z}$ score $<-3.0)$. He was afebrile, pale, dyspnoeic and had subcostal, intercostal and suprasternal retractions with heart rate $110 / \mathrm{min}$ and capillary filling time $<2 \mathrm{sec}$. Respiratory rate was $66 /$ min. with bilateral wheeze. Abdomen was distended with liver being $3 \mathrm{~cm}$ palpable below right costal margin with span of $6.5 \mathrm{~cm}$. Other systems were normal.

Investigations revealed: haemoglobin of $11.6 \mathrm{~g} / \mathrm{dl}$, total leukocyte count $12,200 / \mathrm{mm}^{3}$, with $39 \%$ polymorphs and $51 \%$ lymphocytes. Review of serial previous X-rays showed prominent pulmonary vascularity. Mantoux test was negative. USG abdomen showed small target/granulomatous lesions in liver. USG of cranium and Echocardiography was normal. Blood culture showed no growth. Liver biopsy and high-resolution computed tomography thorax and abdomen couldn't be done due to non availability of these facilities at that time.

Child was initially treated for pneumonia with injection ceftriaxone and gentamycin without any improvement. In view of mother's chest X-Ray being suggestive of pulmonary tuberculosis, child's symptomatology beginning from $3^{\text {rd }}$ week of life, target lesions in liver on USG with failure to thrive possibility of congenital tuberculosis was considered \& patient was started on 4 drug Antituberculosis treatment (ATT) viz. isoniazide, rifampicin, ethambutol and pyrizinamide (HREZ). Following this child condition initially improved with progressive weight gain, improved appetite and activity.

Correspondence to: Lokesh Tiwari, Assistant Professor of Pediatrics, All India Institute of Medical Sciences Patna, India, Tel: +919631638095; Email: lokeshdoc@yahoo.com.

Key words: Congenital tuberculosis, Anti-tuberculosis treatment, paradoxical reaction

Received: July 10, 2017; Accepted: August 18, 2017; Published: August 21, 2017 
However after 2 weeks of ATT child showed sudden clinical worsening in form of fever, lethargy, decreased appetite and increased respiratory distress. For possibility of hospital acquired infection antibiotic was upgraded to injection piperacillin and tazobactum. However no supportive laboratory evidences for the same could be found. When no improvement was noticed, possibility of tuberculous paradoxical reactions (PR) was considered and steroid was started. Following this child responded in form of resolution of fever, decreased respiratory distress and persistent weight gain. Patient is in our regular follow-up and is thriving well and repeat USG abdomen after 2 months of ATT showed clearance of these granulomatous hepatic lesions. Steroid was tapered off after 6 weeks and ATT was continued for $1 \mathrm{yr}$ (2HREZ + 10HR).

\section{Discussion}

Tuberculous bacillemia during pregnancy may result in infection of the placenta or the maternal genital tract $[4,5]$. Congenital tuberculosis is defined when tubercular infection is transmitted to the foetus either haematologically via the umbilical vein or by infected amniotic fluid, which is ingested or aspirated in utero, or during delivery [6]. It should be distinguished from acquired neonatal tuberculosis, where the infant is infected after birth by a contagious adult. Our patient had granulomatous lesions in liver which could not be explained by acquired neonatal tuberculosis.

Diagnostic criteria for congenital tuberculosis were laid down by Beitzki in 1935 and subsequently were revised by Cantwell in 1994 [7]. Singh et al. [8] in 2007 suggested laboratory and clinical findings that may suggest congenital TB, which include a newborn from a TB endemic area with unresponsive worsening pneumonia, a mother with TB and a baby with nonspecific symptoms and the presence of hepatosplenomegaly and fever. Our case, from a TB endemic region, having an unresponsive worsening pneumonia associated with hepatomegaly, with a history of TB in mother, meets the above criteria. Signs and symptoms in congenital TB appears after the first 3 weeks of life at a median age of 28 days (range: 1 to 84 days) [7], index case became symptomatic during $3^{\text {rd }}$ week of life.

Congenital tuberculosis is particularly difficult to diagnose since the non-specific presenting signs and symptoms such as respiratory distress, hepatosplenomegaly, jaundice, fever, lymphadenopathy, lethargy and failure to thrive are also seen in other non tubercular infections.

After 2 weeks of ATT patient showed sudden deterioration and it was difficult to rule out hospital acquired infection (HAI) as patient was still in PICU. He was treated for HAI however; laboratory evidences for HAI were negative. When no improvement was noticed possibility of TB-PR was considered.

Occurrence of PR in HIV-negative patients with pulmonary TB is reported to be $2.4 \%$ [9], where as in patients of extrapulmonary $\mathrm{TB}$ it is much higher ranging from 16 to $50 \%$ [10]. Paradoxical response is defined as the clinical or radiological worsening of pre- existing tuberculous lesions or the development of new lesions not attributable to the normal course of disease in a patient who initially improves with antituberculosis therapy and in whom the onset of paradoxical response is at least 2 weeks after the initiation of treatment [9]. When ATT is started an immune rebound may occur, probably due to the release of $\mathrm{M}$. tuberculosis antigens during the destruction of infected macrophages, which may explain the worsening of clinical symptoms. This clinical entity can be misdiagnosed as superimposed infections, treatment failure, or TB relapse. Our patient having initial improvement with ATT had clinical worsening after 2 weeks with no evidence of secondary infection or treatment failure fulfils these criteria for tuberculosis PR. This is only the $2^{\text {nd }}$ case to be reported of TB-PR in congenital tuberculosis.

Congenital TB is a rare entity and is uniformly fatal if untreated. Treatment of the infant should begin as soon as the diagnosis is suspected without waiting for laboratory confirmation, while appropriate specimens should be obtained fast for bacteriological and histological examination [2]. Our case highlights the importance of keeping high index of suspicion for congenital TB especially in tuberculosis endemic area even in absence of bacteriological isolation and timely institution of ATT.

In case of unexplained clinical or radiological worsening after initial improvement with ATT with no evidence of secondary infection, poor drug compliance or treatment failure, TB-PR should be suspected and steroid considered as this could be life saving for these patients.

\section{References}

1. Smith KC (2002) Congenital tuberculosis: a rare manifestation of a common infection. Curr Opin Infect Dis 15: 269-274. [Crossref]

2. G. Hassan, W.Qureshi, and S. M. Kadri, "Congenital tuberculosis," JK Science, vol. 8, no. 4, pp. 193-194, 2006.

3. Ji Ae Park, Seong Shik Park, Su Eun Park A. paradoxical reaction during antituberculosis therapy for congenital tuberculosis. International Journal of Infectious Diseases, Volume 13, Issue 5, e279 - e281

4. Smith MHD, Teele DW (1990) Tuberculosis. In: Remington JS, Klein JO, eds Infectious diseases of the fetus and newborn infant. 3rd ed. Philadelphia: W.B. Saunders: 834-47.

5. Vallejo JG, Starke JR (1992) Tuberculosis and pregnancy. Clin Chest Med 13: 693707. [Crossref]

6. Miller FJW. Tuberculosis in children. Evolution, Epidemiology, Treatment, Prevention, 1st ed. New Delhi: Churchill Livingstone, 1982, 220-224.

7. Cantwell MF, Shehab ZM, Costello AM, Sands L, Green WF, et al. (1994) Brief report: congenital tuberculosis. N Engl J Med 330: 1051-1054. [Crossref]

8. Singh M, Kothur K, Dayal D, Kusuma S (2007) Perinatal tuberculosis a case series. $J$ Trop Pediatr 53: 135-138. [Crossref]

9. Cheng VCC, Ho PL, Lee RA, Chan KS, Chan KK, et al. (2002) Clinical spectrum of paradoxical deterioration during antituberculosis therapy in non-HIV-infected patients. Eur J Clin Microbiol Infect Dis 21: 803-809. [Crossref]

10. Hawkey CR, Yap T, Pereira J, Moore DA, Davidson RN, et al. (2005) Characterization and management of paradoxical upgrading reactions in HIV-uninfected patients with lymph node tuberculosis. Clin Infect Dis 40: 1368-1371. [Crossref]

Copyright: (C2017 Tiwari L. This is an open-access article distributed under the terms of the Creative Commons Attribution License, which permits unrestricted use, distribution, and reproduction in any medium, provided the original author and source are credited. 